\title{
Automatic Satellite Image Enhancement Approach to Extract the Features Based on a Morphological Building Index
}

\author{
K. Priyanka ${ }^{1}$, Uppada Srikanth ${ }^{2}$, Shaik. Shahensha Khader ${ }^{3}$ \\ ${ }^{1}$ Department of ECE, Sri KS Raju Institute of Technology and Sciences, Moinabad, TS, 501504 -India \\ ${ }^{2}$ Department of ECE, ANURAG College of Engineering, Ghatkesar, TS, 501301-India \\ ${ }^{3}$ Department of ECE, Mannan Institute of Science and Technology, Nyalata, TS, 501503-India
}

\begin{abstract}
The satellite imagery and its importance are growing in the modern digital image processing. The implementation of the enhancement techniques in the satellite image processing is commonly observed practice and a new investigation is carried out in this study to investigate the morphological characteristics and the sensitivity of contrast-based textural measurements to derive the high resolution satellite imagery. The investigation is done after the deployment of the diverse image enhancement techniques to get the statistical analysis and the idea of the proposed study is to build an application framework for the detection of the built-up/non built in reluctant manner. The earlier method utilizes a low-resolution reference layer which works based on the supervised learning and the approach is successful in reducing the uncertainty and has ability to optimize the quality of the reference layer. The goal is to achieve the best computation of the contrast-based textural/morphological features by adjusting the image histogram in a suitable manner. An optimized case study is presented in this work to test the image enhancement operations such as linear and de-correlation stretching. The performance of the image enhancement operations is tested through ROC and compared with the earlier methods the proposed work achieves better performance and high efficiency.
\end{abstract}

Keywords: Satellite imagery, High resolution, Feature extraction, Morphological characteristics, contrast-based textural measurements, Image histogram, SVM, Image enhancement

\section{Introduction}

The acquisition of the digital content (Object) by using the digital sensor and the acquired digital content perception on a digital screen is termed as the digital image processing. The digital image processing implementation in the prominent research fields such as security, medicine, satellite imaginary, computer vision, military, transport, genetics, sonar applications, etc. has introduced the digitalization to visualize the digital content in a pleasant manner.

The satellite imagery is an important aspect in the digital image processing and it is vividly used to detect the location based on the latitudes and longitudes as seen in applications like Google maps, Apple maps, and defense, to get the space related information which helps to understand it in depth manner. The removal of the noise from the satellite images is the most challenging task and the research work must be done in depth way. The research work reported in the literature is useful to reduce the impact of the noise content from the rest of the image, but there is a chance of losing the significant data.

The image enhancement techniques popular in the satellite image processing are contrasted adjustment, de-correlation stretching, histogram equalization, and adaptive filtering belongs are the methods which are used to enhance the satellite images. The above-mentioned enhancement techniques are classified as pixel/spatial-based approaches and in real time it has alternative approaches such as Fourier decomposition, wavelet transform, and discrete cosine transform. The techniques are quite useful in improving the visual image quality and manual parameter training is usually involved with it.

The consistency has been a problem in the un-calibrated images and the main goal of this study is to find an automatic standardization of the un-calibrated images in an accurate manner. The study presented here is to testify the sensitivity of contrast-based textural measurements and on the other end it focuses to perform the computation of the morphological characteristics over various gray models. Another important aspect is to find the alternatives which might able to create the suitable environment for conducting standardized feature extraction with reliability. The satellite imagery based feature extraction is a research area to understand the image content in a statistical way, but existing works proposed in the literature reveal a fact that the feature extraction alone can't give total features which need to understand the satellite imagery in total. The proposed study finds an ample solution to extract the total information by constituting a data preparation just before the feature extraction implementation. The feature extraction is followed by data preparation concept which helps to maintain the quality of the textural/morphological characteristics with a low computational burden which might be considered as a better option than the traditional methods in understanding the satellite imagery.

\section{Related Data}

\section{A. Image enhancement}

The digital images are dominating the modern world with the introduction of the digital concept in popular domains and the introduction of the digital concept has both 


\section{International Journal of Science and Research (IJSR) \\ ISSN (Online): 2319-7064}

Index Copernicus Value (2015): 78.96 | Impact Factor (2015): 6.391

advantages and disadvantages in their respective manner. The degradation of the image quality has various reasons and the main reasons are classified into two categories, namely man-made errors (handshake) and environmental issues (abnormal lighting conditions, fog and so on). The digitally stored digital content is termed as a digital image and its manipulation is observed by the human visual system and the observed manipulated data is processed in computer graphics to improve the digital content quality with the help of software. The process of converting the abnormal form of content to the normal form is done by processing the contrast and illumination to provide the best visual quality and the software using for processing the digital content has ability to support various filtering techniques for altering the images in various ways.

\section{Principle objective of image enhancement}

The digital image processing has shown its presence in all research fields to support various applications and the applications based on digital image processing are increasing in a large way to meet the criteria of the modern world requirements. The image quality must be good so that the human visual system can perceive the image information in hassle-free manner. The noise affected or noise related contents affected digital contents need to be enhanced to make the perception of the digital content in a pleasant way and the processing of the image can be done in an effective manner by the introduction of the image enhancement methods. The digital image processing comprises of images, videos, graphics, etc. and the implementation of the enhancement method has ability to improve the edges, boundaries and display related issues. The introduction of the enhancement technique does have ability to increase the inherent information, but it has ability to increase the detection of the dynamic range of the chosen features.

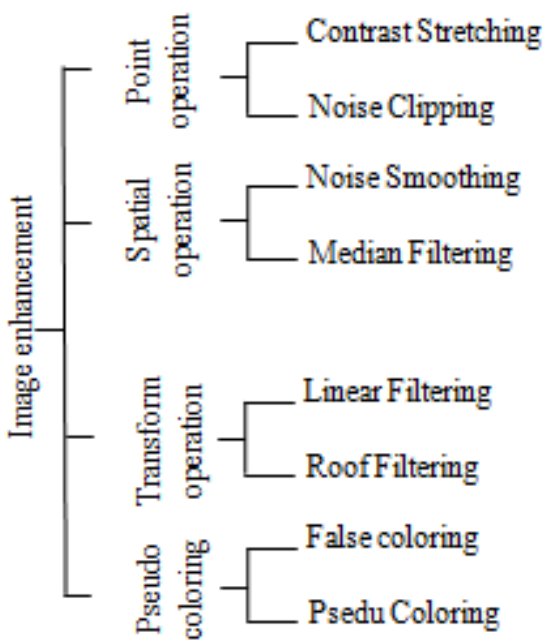

Figure 1: Image enhancement operations

The image enhancement is popular in the processing of the digital content and also attain the satisfactory results in various applications. The quantifying remained as the most challenging aspect in the image enhancement and the desired results can be achieved in the experimental results only after the collaboration of the interactive procedures with enhancement techniques and this collaboration is mandatory because a large number of enhancement methods are empirical. The enhancement techniques are classified into two categories as

(i) Spatial domain: The direct manipulations of the image pixels on the image plane are defined as the spatial domain

$$
g(x, y)=f(x, y) * h(x, y)
$$

(ii) Frequency domain: The enhancement of the image performed based on the linear and convolution operations is defined as the frequency domain.

$$
G\left(w_{1}, w_{2}\right)=F\left(w_{1}, w_{2}\right) H\left(w_{1}, w_{2}\right)
$$

\section{B. Support Vector Machine (SVM)}

SVM will maximize the margin around the separating hyper plane. Then the decision function is specified by a subset of training samples those are called support vectors. There is a problem of minimization and maximization of variables. It will follow the text classification method.

\section{History}

- Pre 1980's: The learning methods which are used before 1980 are followed linear decision surfaces. The properties which are used in linear learning methods are theoretically good.

- 1980's: To learn the non-linear decision surfaces decision trees and NNs are used. Decision trees are one way to display an algorithm. NNs (neural networks) are used to represent a complex relationship between input and output. These two methods are efficient in 1980's, but theoretical basis are less.

- 1990's: In 1990's to solve the non linear functions computational learning theory is developed. Computational learning theory will perform the design and analysis of machine learning algorithms. By using this method we can get the good theoretical properties.

- Two independent developments within last decade:

1. Computational learning theory

2. Kernel functions by using this we can get the satisfactory results, but in real time it is not good. Then we are going for statistical learning theory.

\section{(i) Statistical learning theory}

In this the data can be analyzed by mathematically. The difference between the input and output can be known by statistical values, by this we can predict some features of future data. Accuracy can be measured by using this theory. Basic idea of support vector machines

- For linear separable planes we can give the satisfactory results of hyper plane.

- The data is not linearly separable by using the transformation techniques to then extend it to patterns kernel function.

\section{(ii) Features of SVM and kernel methods}

These two methods mainly based on theoretical model of learning. Theoretical performance will be perfect in these methods. The data can be divided into parts and then it will design their components. These methods are not affected by local minima. There is no problem of dimensionality.

\section{(iii) Support vectors}

Support vectors means data points that lie closest to the decision surface. The classification can be very difficult to 


\section{International Journal of Science and Research (IJSR) \\ ISSN (Online): 2319-7064}

Index Copernicus Value (2015): 78.96 | Impact Factor (2015): 6.391

process. The support vectors are having connection with decision surface for the satisfactory location. We can show the perfect hyper plane stems from the function class with the lowest capacity.(VC dimension)

\section{Problem Statement}

The satellite image processing is highly demanded research field in the modern world. The secrets about the universe, navigate the different locations on planet earth and under the earth such as detecting the coal regions, various defence operations and finally various other operations related to weather reports, academia, mapping, remote sensing, etc. The various issues frequently occur in the satellite images area as follows

- The perception of the satellite image is a challenging task as its resolution is in the form of 16 bit supported while normal user defined gadgets like mobiles, laptops and televisions are 8 bit supported. The usage of tone mapped algorithms can effectively solve the issue in the accurate way.

- The image content acquired using the satellites is in high resolution with good quality at initial stages, but at the time of its reception at earth it loses its high quality and remained as the low resolution image. The perception and feature extraction is challenging task in satellite image processing.

- The above metioned problems are resolved using the high-resolution satellite imagery approach for maintaining the relevant quality levels and contrastbased textural/morphological features in the proposed study. The goal is to achieve the best computation of the contrast-based textural/morphological features by adjusting the image histogram in a suitable manner

\section{Proposed Method}

\section{A. Image features}

The image features poses great importance in statistical analysis and the estimation of the textural measurements has the ability to give the detailed statistical analysis. The textural measurements can be obtained by performing the estimation between the contrast pixel intensity and its neighbors through Haralick's measurement. A textural layer named PANTEX is formed by fuzzy composition factors such as quantization, length and orientation. Another interesting aspect included in this study is the inclusion of the recently introduced morphological operations named MBI (morphological building index) and the resultant outcome of the MBI is accurate in terms of brightness, size, contrast, directionality, and shape. The novelty of the MBI operation is based on the integration of the multi-scale and multi-directional morphological operators which are used to indicate the building characteristics in an accurate manner.

The image feature extraction in earlier methods purely depends on the statistical learning and training samples while the proposed PANTEX and MBI approaches are automatic indices whose operations are operated in an automatic way. The presence of the high dimensionality is the other problem which frequently occurs in the existing works and it is successfully overcome by introducing the automatic indices in place of manual indices and the reduction of the high dimensionality by introducing the cooccurrence matrix computation and the further progress is carried out by converting the multi-scale to 8-bit grayscale images.The contrast adjustment is another approach followed in the satellite imagery by which issues related to morphological operations are resolved by adjusting the image intensities.

\section{B. Algorithm description}

The description of the proposed algorithmic approach is as follows

1) A popular classification approach named SVM is used in this algorithm for better classification results. The SVM classifier is a binary classifier which is trained to operate in the light of a low-resolution reference layer.

2) The estimation of the built-up (BU) and nonbuilt-up (NBU) is the interesting thing carried out in this algorithm. The optimal hyperplane is utilized to separate the built-up (BU) and nonbuilt-up (NBU) classes through nonlinear mapping

3) The modification of the reference layer class labels through ad hoc treatment is highly useful to obtain the respective training samples and has the ability to delimit the hyperplane into a high-dimensional feature space.

4) Each class is treated in an individual way by adjusting the histogram adjustment which helps to create the favorable environment for the extraction of the textural measurements.

\section{Syllogism}

Support vectors (SVs) are the closest tuples of measurements to the hyperplane with respect to $\mathrm{H}$; consequently, they contain the critical information for the class separation. In our application, having as fact that the reference layer does not constitute an accurate template mainly due to its low spatial resolution, the meaning of SVs matches with the concept of uncertainty that is inherent along the class boundaries. Thereafter, three options are deemed for the SVs usage.

1) To totally remove their respective class labels from the reference layer: this decision targets at the increase in both intra-class similarity and inter-class dissimilarity; however, it has high risk due to the loss of potentially useful information for the discrimination of the classes.

2) To set the SVs of BU class to the NBU class and change accordingly the respective class labels in the reference set: this is a biased decision having its basis on the fact that when the reference layer is projected into the spatial resolution of the input image $(2.5 \mathrm{~m})$, it represents an optimistic version of the BU reality: it introduces commission errors by having pixels in BU class, while the corresponding spectral signature fits better with NBU class.

3) To remove all SVs and build another SVM by using the remaining vectors as training set: the hypothesis behind this choice is that the second batch of SVs is expected to have fewer and more reliable vectors, i.e., to act as a confidence set for the separation of the two classes.

\section{Reference Sets Generation}

LetIm $\times \mathrm{n} \times 3$ be the input image with $\mathrm{m}$ and $\mathrm{n}$ denoting the number of rows and columns, respectively, per image band. 


\section{International Journal of Science and Research (IJSR) \\ ISSN (Online): 2319-7064}

Index Copernicus Value (2015): 78.96 | Impact Factor (2015): 6.391

Next, is downsampled to the spatial resolution of the reference layer using interpolation and the average operator; it becomes $\mathrm{X}=[\mathrm{bNIbR} b \mathrm{~b}] \mathrm{k} \times 3=[\mathrm{xi}=1, \ldots, \mathrm{k}$ ]with column vectors $b$ NI, $b$ R, and $b$ G denoting the bands near infrared, red, and green, respectively ; $\mathrm{xi}$ signifies triplets and $\mathrm{k}$ denotes the number of pixels per band. The downsampling saves much of the computational burden to compute the kernel/similarity matrix required by the SVM [training complexity $\mathrm{O}(3 \mathrm{k} 2)]$. The binary reference layer is represented by the vector $\operatorname{SSL}$ (1) $\mathrm{k}=[\mathrm{yi}=1, \ldots, \mathrm{k}]$, yi $\in\{-1,1\}$, where -1 refers to the class NBU and 1 to the class BU. The superscript of SSL notation indicates the version of the reference layer and the subscript denotes the number of pixels.

The control variable $\mathrm{C}>0$ is used to penalize classification errors during training. The solution of (1) leads to the definition of the discrimination function as follows

$$
\begin{aligned}
S S L_{k}^{(2)} & =\left\{\begin{array}{c}
y_{j}, j \notin V \\
0, \text { otherwise }
\end{array}\right. \\
S S L_{k}^{(3)} & =\left\{\begin{array}{c}
y_{j}, j \notin V \\
-1, \text { otherwise }
\end{array}\right.
\end{aligned}
$$

Build a new SVM by using $X=[x j] \forall j / \in$ Vas training set and define the respective discrimination function. Subsequently, utilize this function to classify the pixels of the original imageImn $\times 3$. The resulting binary image SSL (4) $m \times n$ becomes the new reference. Actually, it represents the downscaled version of SSL (1) Note that the number of SVs derived from the second SVM is $30-40$ times less than the number of the first SVs. This reduces a lot the classification complexity that scales linearly with the number of SVs.

The following listing describes the generation of the alternative reference images and the downscaling method using a type of pseudocode

\section{E. Algorithm flow}

Algorithm 1: Generate Reference Images Pseudocode

1: I $\leftarrow$ Input multiband image

$2:$ Ref $\leftarrow$ Binary image $(-1,1)$ used as reference

$3: \mathrm{X} \leftarrow$ Downsample(I) according to Ref dimensions

4: Train SVM(X, Ref)

$5: \mathrm{SV}=\left\{\boldsymbol{x}_{v}: v \in V\right\}$

6: Generate new reference $\mathrm{y} \leftarrow$ Ref:

option(i): Set 0 to the pixels of $Y$ that correspond to the support vectors of SV

option(ii): Set -1 to the pixels of $\mathrm{Y}$ that correspond to the support vectors of SV

option(iii): $\operatorname{Train} S V M\left(X / S V, Y\left\{y_{j}, j \in V\right\}\right)$

$S V_{1}=\{$ new support vectors $\}$

$\mathrm{Y}=$ SVMclassify $\left(\mathrm{I}, S V_{1}\right)$

\section{Output Y}

\section{Results and Analysis}

original image

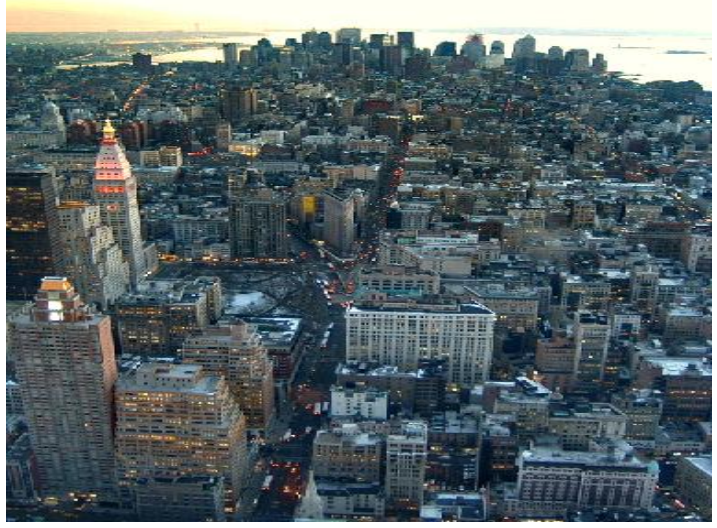

Figure 1: A low resolution input image

Analysis: A low resolution satellite image is taken as input. The feature extraction from the low resolution image is remain as the challenging task due to its abnormal intensity levels. This image used for further processing using the proposed method to yield a higher resolution image as an outcome.

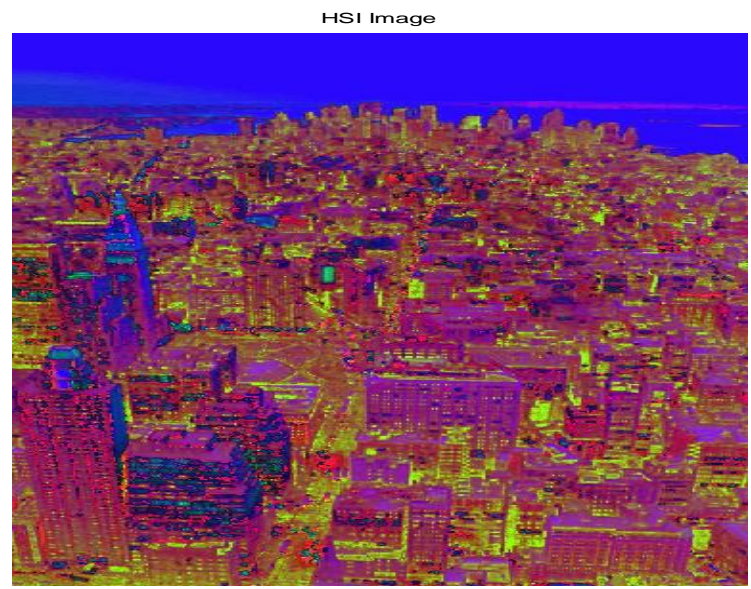

Figure 2: HSI Image

Analysis: The color spaces is the important aspect in the digital image processing and using it HVS can have ability to perceive the data in a pleasant manner. The RGB color space is a basic color space and won't have the ability to fulfill the basic needs as the user desired and the conversion of RGB to HIS makes a way to process the image in terms of the color as shown in the above figure.

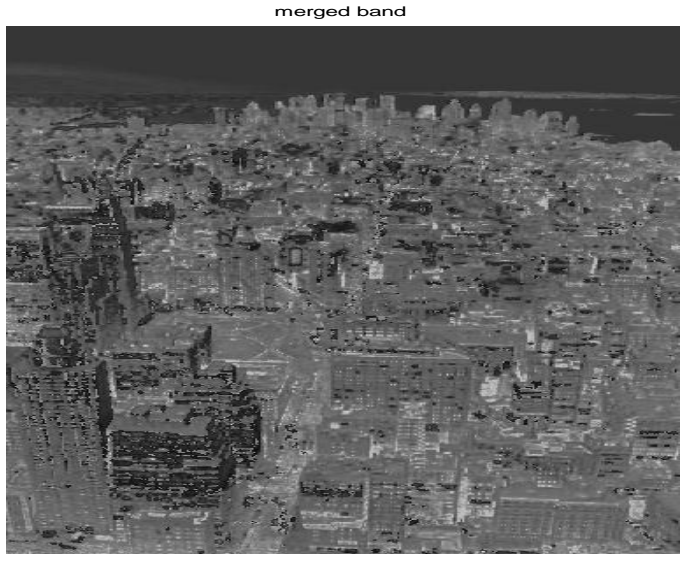

Figure 3: Merged band image 


\section{International Journal of Science and Research (IJSR) \\ ISSN (Online): 2319-7064 \\ Index Copernicus Value (2015): 78.96 | Impact Factor (2015): 6.391}

Analysis: The merged band image is the innovative concept proposed in this paper where the perception of the digital images is done in specialized format as shown in above figure. The above image is the combination of 7 bands of the image to improve the quality and contrast of an image.

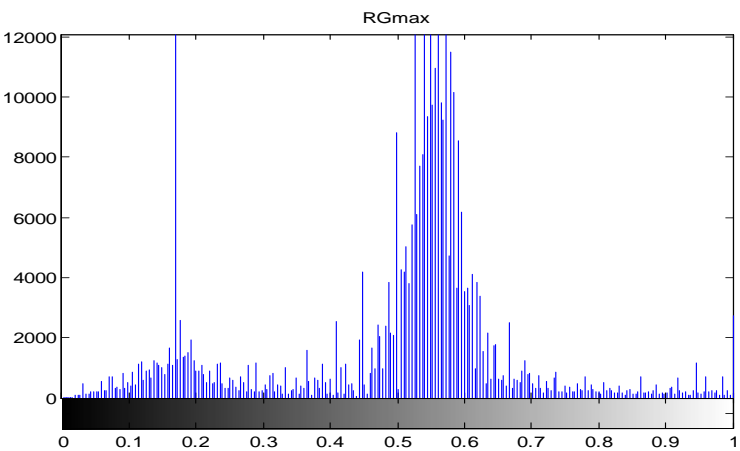

Figure 4: Image histogram and contrast stretching of $R G_{\max }$

Analysis: The image statistics cannot be perceived by the human visual system and its graphical representation, i.e. histogram has the ability to make the perceptibility of image statistics in terms of bins. The $R G_{\max }$ in the above figure refers to the maximum decomposition of the multichannel image. The histogram analysis of grayscale and red color space is performed.

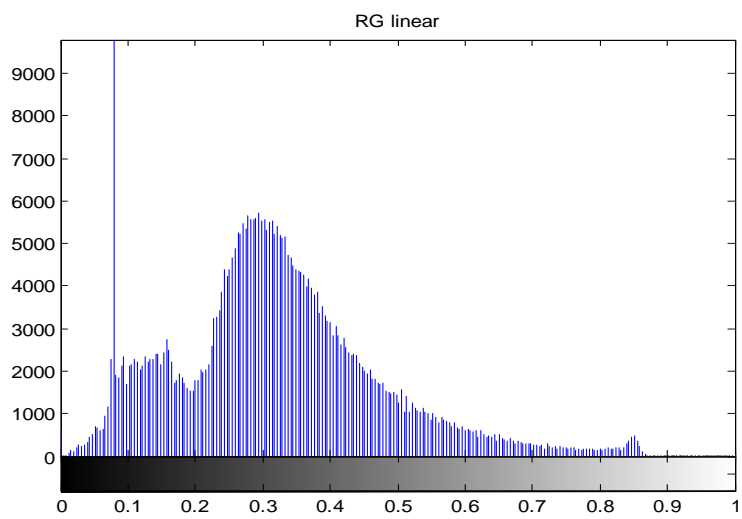

Figure 5: Image histogram and contrast stretching of $R G_{\text {linear }}$

Analysis: $R G_{\text {linear }}$ refers to the weighted average of gammacorrected RGB. The histogram analysis of both red and green component is evaluated based on the reference band $\mathrm{O} 2$.

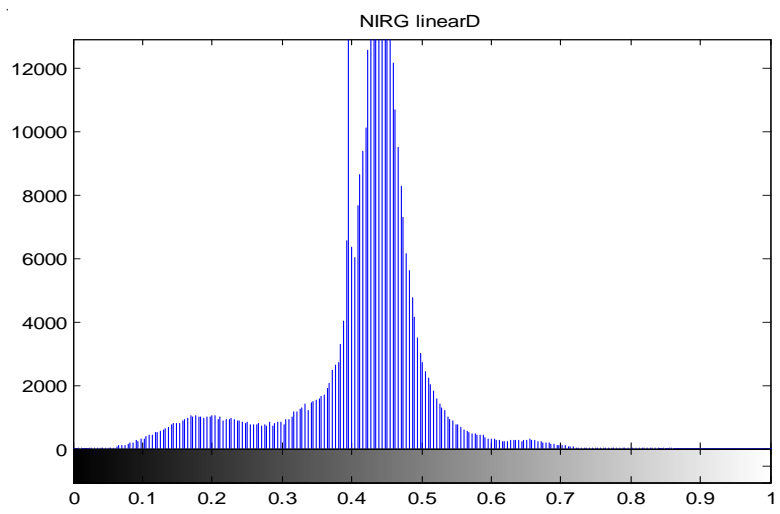

Figure 6: Image histogram and contrast stretching of $N I R G_{\text {linearD }}$
Analysis: The above figure shows the histogram analysis of three components(InfraRed, red and green) with reference to the band $(\mathrm{O} 3)$.

\section{downsampled image}

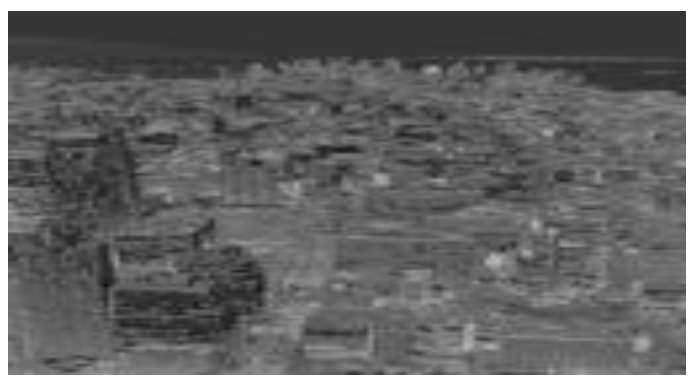

Figure 7: Downsampling of a low resolution image.

Analysis: Downsampling an original reference band to reduce the sampling rate. The low resolution is not in the normal way to ready for extraction of the features so the downsampling on it is considered as the mandatory step.

\section{histogram streching image}

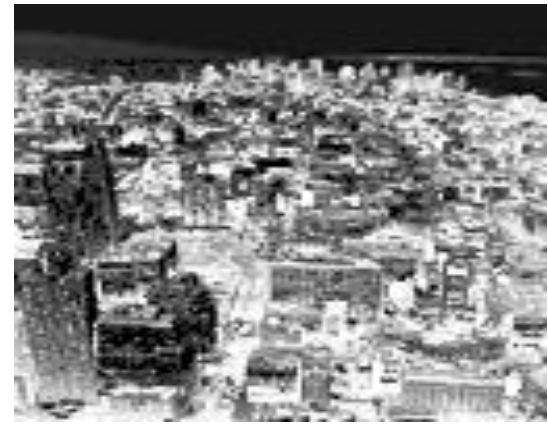

Figure 8: Histogram stretching image

Analysis: The digital image examination is done in accurate manner by histogram and enhancement process first uses the histogram before perform any task of image enhancement which helps in yielding the best quality image. The stretching of the image is done and its outcome is shown in above figure
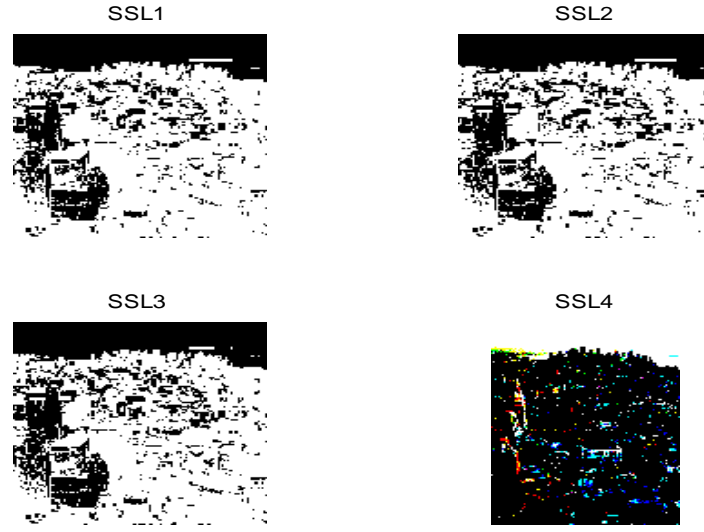

Figure 9:SSL1,SSL2,SSL3,SSL4 are the four bands of a reference image

Analysis: The four bands of the reference is shown in the above figure in different segments starting from SSL1 to SSL4 for better results 


\section{International Journal of Science and Research (IJSR) \\ ISSN (Online): 2319-7064}

Index Copernicus Value (2015): 78.96 | Impact Factor (2015): 6.391
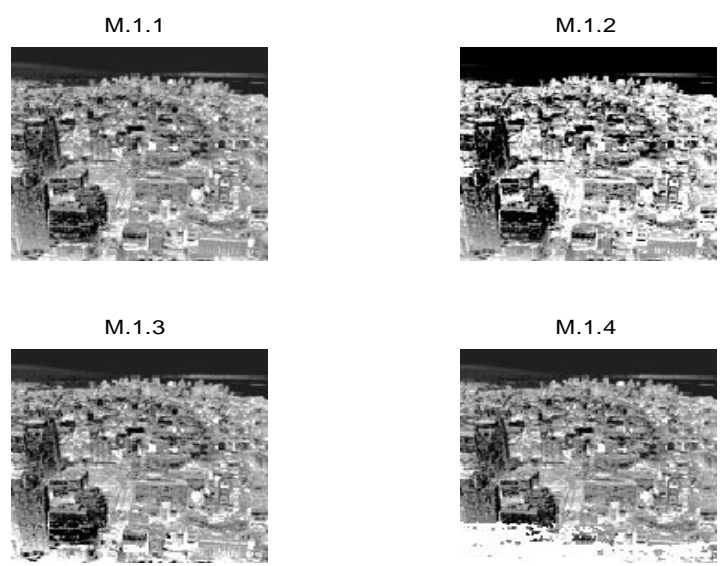

Figure 10: The contrast enhancement of reference image at mode1

Analysis: Image contrast enhancement is the predominant fundamental step in the image processing and digital image enhancement creates an image which is perceived by the human visual system (HVS) in a pleasant way.
M.2.1

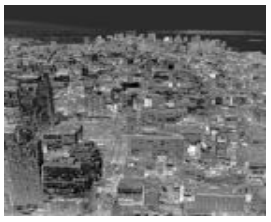

M.2.3

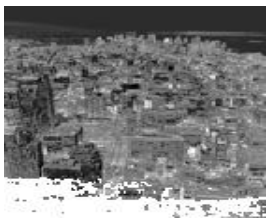

M.2.2

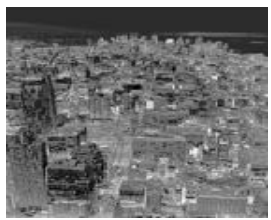

M.2.4

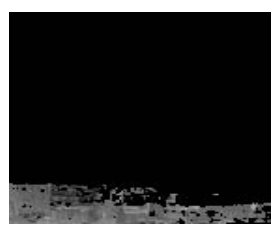

Figure 11: The contrast enhancement of reference image at mode1

Analysis: The contrast enhancement makes the perception of the image in the best way and in the proposed study it is represented in terms of $\mathrm{M}$ level as shown in the above figure.

\section{Conclusion}

The satellite image perception through digital displays remain as the unresolved issue in the satellite imagery as the content is in low resolution which makes the feature extraction a difficult one. The proposed method performs the intense research on the previous approaches and came up with innovative solutions with good accuracy. The usage of the high-resolution satellite imagery approach for maintaining the relevant quality levels and contrast-based textural/morphological features for better accuracy than the earlier methods. The earlier methods fail to process on the both resolution and feature extraction while the proposed done it with ease.

\section{References}

[1] F. Y. Shih, Image Processing and Pattern RecognitionFundamentals and Techniques. Hoboken, NJ, USA: Wiley, 2010.

[2] R. C. Gonzalez and R. E. Woods, Digital Image Processing, 2nd ed. Englewood Cliffs, NJ, USA: Prentice Hall, 2002.

[3] S. Kim, W. Kang, E. Lee, and J. Paik, "Wavelet-domain color image enhancement using filtered directional bases and frequency-adaptive shrinkage," IEEE Trans. Consum. Electron., vol. 56, no. 2, pp. 1063-1070, May 2010.

[4] A. R. Gillespie, A. B. Kahle, and R. E. Walker, "Color enhancement of highly correlated images. I. Decorrelation and HSI contrast stretches," Remote Sens. Environ., vol. 20, no. 3, pp. 209-235, Dec. 1986.

[5] H. Demirel, C. Ozcinar, and G. Anbarjafari, "Satellite image contrast enhancement using discrete wavelet transform and singular value decomposition," IEEE Geosci. Remote Sens. Lett., vol. 7, no. 2, pp. 333-337, Apr. 2010.

[6] G. Srilekha, V. K. Kumar, and B. Jyothi, "Satellite image resolution enhancement using DWT and contrast enhancement using SVD," Int. J. Eng. Res. Technol. (IJERT), vol. 2, no. 5, pp. 1227-1230, May 2013.

[7] [Online].

Available: http://cidportal.jrc.ec.europa.eu/copernicus/services/ webviewer/core 003

\section{Author Profile}

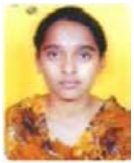

K. Priyanka received the bachelor of engineering in electronics and communication engineering from ISL Women's Engineering College, Hyderabad, Telangana State, India, in 2014, the M.Tech. degree in electronics and communication engineering from Sri KS Raju Institute of Technology and Sciences, Moinabad Mandal, Hyderabad, Telangana, India, in 2016. She is working as the MATLAB programmer in the Krest technologies from past two years and her research interests include digital image processing, satellite imagery, digital signal processing, wireless communications feature extraction and computer vision

Uppada Srikanth received the Bachelor of Technology degree in electronics and communication engineering from Anurag College of Engineering, Ghatkesar, Telangana, India, Affiliated to JNTU Hyderabad university, in 2015 . He is working as the MATLAB programmer in the Krest technologies from past one year and his research interests include digital image processing, OFDM, Saliency detection, satellite imagery, digital signal processing, wireless communications feature extraction and computer vision

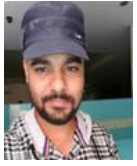

Shaik. Shahensha Khader received the B.Tech. degree in electronics and communication engineering from JNTU Hyderabad university affiliated Mannan Institute of Science and Technology college. $\mathrm{He}$ is working as the MATLAB programmer in the Krest technologies from past three years and his research interest include digital image processing, Content based image retrieval, Feature extraction, OFDM, Saliency detection, satellite imagery, digital signal processing, wireless communications feature extraction and computer vision 